Check for updates

Cite this: RSC Adv., 2019, 9, 37287

Received 29th September 2019 Accepted 8th November 2019

DOI: 10.1039/c9ra07911d

rsc.li/rsc-advances

\section{Isomerization enabling near-infrared electron acceptors $\uparrow$}

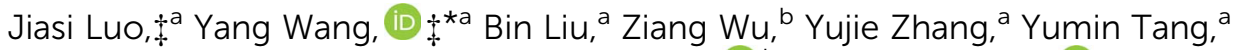 \\ Peng Chen, ${ }^{a}$ Qiaogan Liao, ${ }^{a}$ Han Young Woo (DD ${ }^{b}$ and Xugang Guo (DD *a
}

An isomerization method was utilized to yield a novel near-infrared nonfullerene acceptor DTA-IC-M. By simply changing the linking fashion between the anthracene and neighboring thiophenes, a remarkable redshift $(\sim 170 \mathrm{~nm})$ of absorption was observed from DTA-IC-S to its isomer DTA-IC-M which shows a maximum absorption peak over $800 \mathrm{~nm}$ with a narrow bandgap of $1.35 \mathrm{eV}$. Due to the enhanced photo-to-current response in the near-infrared region, an improved short-circuit current of $12.96 \mathrm{~mA}$ $\mathrm{cm}^{-2}$ was achieved for the DTA-IC-M based OSCs.
Nonfullerene n-type organic semiconductors have attracted increasing attention ${ }^{1}$ as electron acceptors in organic solar cells (OSCs) owing to their favorable features, such as widely tunable absorption windows and controllable energy levels. ${ }^{2}$ It is well known that the majority of solar irradiation is located at nearinfrared (NIR) region (>750 nm), hence exploring NIR nonfullerene acceptors (NNAs) is highly desirable in order to achieve efficient OSCs with high short-circuit current $\left(J_{\text {sc }}\right)$. In addition, NNAs will be beneficial for realizing highperformance semitransparent and tandem OSCs. For example, a carbon-oxygen-bridged NNA reported by Ding et al. shows an ultralow bandgap $\left(E_{\mathrm{g}}\right)$ of $1.26 \mathrm{eV}$ with the maximum absorption peak over $800 \mathrm{~nm}$, which enables a remarkable $J_{\mathrm{sc}}$ of $27.39 \mathrm{~mA}$ $\mathrm{cm}^{-2}$ with a power conversion efficiency (PCE) of $14.62 \%{ }^{3}$ in single-junction OSCs and a much higher PCE of $17.36 \%{ }^{4}$ in tandem OSCs. Recently, Zou et al. invented a novel A-D-A-D-A type NNA with a low $E_{\mathrm{g}}$ of $1.33 \mathrm{eV}$ as well as good electrontransporting ability which boosted the PCE to $15.7 \%$ and $16.5 \%$ in binary $^{1 h}$ and ternary ${ }^{5}$ single-junction OSCs, respectively. Hence, huge potential still exists for the development of NNAs.

Generally, there are three main strategies for designing NNAs as shown in Fig. 1. Taking the simplest fused-ring electron acceptor IDT-IC as the starting point, elongating the $\pi$ conjugation of molecular skeleton is the most widely used

\footnotetext{
${ }^{a}$ Department of Materials Science and Engineering, The Shenzhen Key Laboratory for Printed Organic Electronics, Southern University of Science and Technology (SUSTech), No. 1088, Xueyuan Road, Shenzhen, Guangdong 518055, China. E-mail: wangy6@sustech.edu.cn; guoxg@sustech.edu.cn

${ }^{b}$ Department of Chemistry, Korea University, Seoul 02841, South Korea

$\dagger$ Electronic supplementary information (ESI) available: Experimental details, synthetic procedures, absorption spectra, theoretical calculations, charge mobility measurement, optimal photovoltaic parameters of OPVs, AFM, TEM, GIWAX. See DOI: 10.1039/c9ra07911d

\$ These authors contributed equally to this work.
}

method to redshift absorption (method A). ${ }^{6}$ As a result, a low $E_{\mathrm{g}}$ of $1.35 \mathrm{eV}$ is obtained for NNA1 having a 8-ring fused backbone. ${ }^{7}$ Alternatively, introducing strong electron-donating functionalities such as alkoxy or alkyl amino groups can also reduce $E_{\mathrm{g}}$ (method B), ${ }^{4,8}$ e.g. a similar $E_{\mathrm{g}}$ of $1.34 \mathrm{eV}$ is achieved for NNA2. ${ }^{8 \mathrm{~d}}$ In addition, narrow $E_{\mathrm{g}}$ could be also realized by increasing quinoidal character ${ }^{9}$ as demonstrated in NNA3 (method C), which shows an $E_{g}$ of $1.32 \mathrm{eV} \cdot{ }^{9 b, 10}$ Despite of these achievements in red-shifting absorption and narrowing bandgap for electron acceptors, tedious synthesis and high-cost production are usually required due to the introduction of additional specific functional groups onto the initial molecular backbones. Therefore, the exploration of NNAs with simple structural modification and facile synthesis is preferred for future commercialization of OSCs.

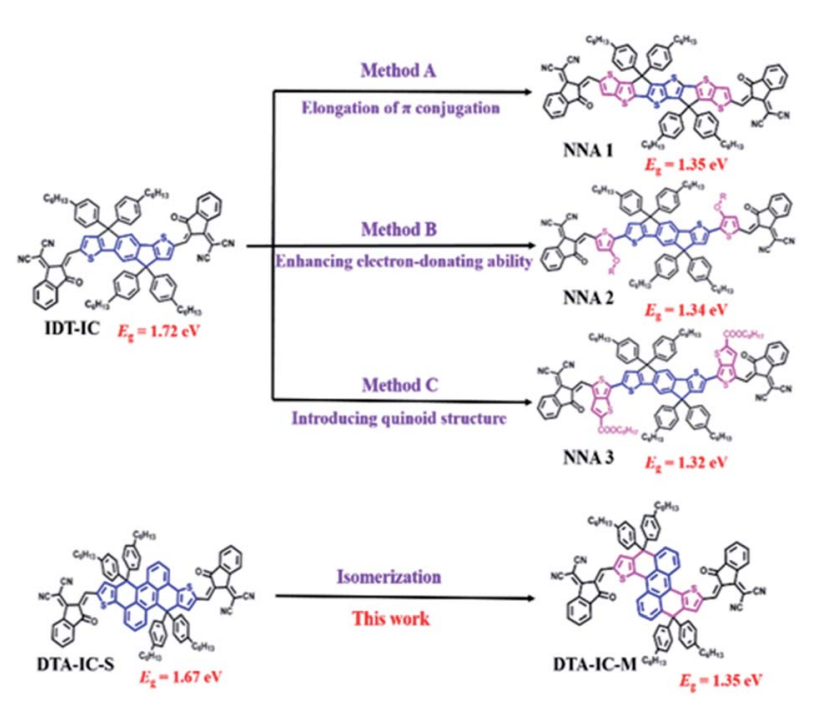

Fig. 1 Typical methods employed to reduce the bandgap $\left(E_{\mathrm{g}}\right)$ of nonfullerene acceptors. 
As reported in our previous work, ${ }^{11}$ polycyclic aromatic hydrocarbon (PAH) based acceptors feature readily tailorable chemical structures and facile synthesis as well as fine-tuned optoelectronic properties. Based on such simple-to-use chemical backbones, here, an isomerization method was employed to produce a novel NNA without increasing the workload of synthesis. As depicted in Fig. 1, we have developed an anthracene based acceptor DTA-IC-S in our previous work, ${ }^{11}$ which possesses a medium $E_{\mathrm{g}}$ of $1.67 \mathrm{eV}$ similar to that of IDT-IC. Interestingly, after simply changing the linking fashion between the anthracene core and fused thiophene units, a significant redshift of nearly $170 \mathrm{~nm}$ could be realized for the newly-developed acceptor DTA-IC-M, rendering an absorption maximum $\left(\lambda_{\max }\right)$ at $822 \mathrm{~nm}$ as well as a low $E_{\mathrm{g}}$ of $1.35 \mathrm{eV}$. Owing to its wide photo-response in NIR region (750-950 nm), an enhanced $J_{\mathrm{sc}}$ of $12.96 \mathrm{~mA} \mathrm{~cm}{ }^{-2}$ could be achieved in the DTA-IC$\mathrm{M}$ based solar cells compared to DTA-IC-S, enabling a PCE of $4.2 \%$.

As shown in Scheme $\mathbf{S 1 , \dagger} \uparrow$ the synthetic route to DTA-IC-M is exactly the same with DTA-IC-S ${ }^{11}$ except for different commercially available starting materials (i.e. 1,5-dibromoanthracene for DTA-IC-S and 9,10-dibromoanthracene for DTA-IC-M), ensuring low-cost and facile synthesis. The structure and purity of new acceptor DTA-IC-M was proved by ${ }^{1} \mathrm{H}$ NMR, ${ }^{13} \mathrm{C}$ NMR, high resolution mass spectrometry, and elemental analysis as included in the ESI. $\dagger$

The UV-vis absorption spectra of DTA-IC-S and DTA-IC-M are shown in Fig. 2a with the corresponding data summarized in Table 1. Surprisingly, a significant redshift of $\lambda_{\max }$ as large as $145 \mathrm{~nm}$ is observed for DTA-IC-M in solution versus its isomer DTA-IC-S, accompanied by an intensified molar extinction coefficient $(\varepsilon)$ from $1.63 \times 10^{5} \mathrm{M}^{-1} \mathrm{~cm}^{-1}$ of DTA-IC-S to $2.23 \times$
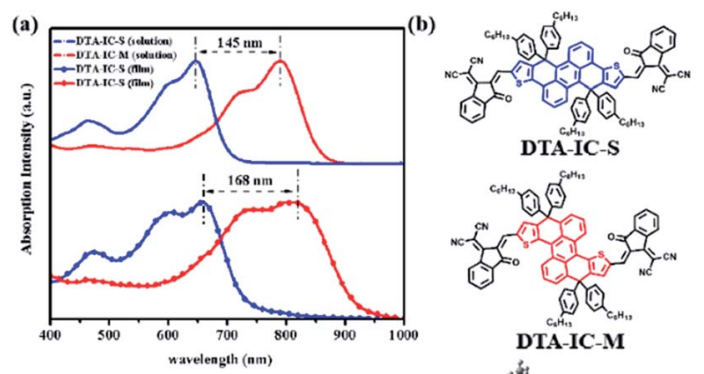

(c)

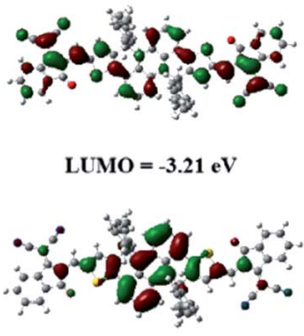

$\mathrm{HOMO}=-5.32 \mathrm{eV}$
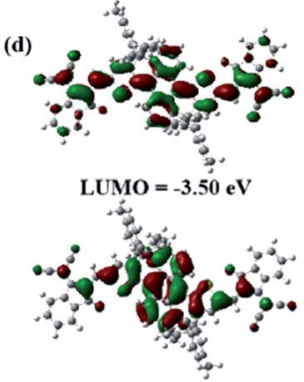

$\mathrm{HOMO}=-5.25 \mathrm{eV}$

Fig. 2 (a) Normalized absorption spectra of DTA-IC-S and DTA-IC-M in $\mathrm{CHCl}_{3}$ solutions and as thin films; (b) chemical structure of DTA-IC$S$ and DTA-IC-M; (c and d) frontier molecular orbitals of (c) DTA-IC-S and (d) DTA-IC-M.

$10^{5} \mathrm{M}^{-1} \mathrm{~cm}^{-1}$ of DTA-IC-M (Fig. S1 $\dagger$ and Table 1). Ongoing from solution to thin film, the redshift of $\lambda_{\max }$ increases to $168 \mathrm{~nm}$ due to enhanced intermolecular interactions in solid state with the $\lambda_{\max }$ of DTA-IC-M reaching $822 \mathrm{~nm}$ in NIR region. As a result, a medium bandgap electron acceptor DTA-IC-S $\left(E_{\mathrm{g}}=\right.$ $1.67 \mathrm{eV})$ could be transformed to a NNA DTA-IC-M $\left(E_{\mathrm{g}}=1.35 \mathrm{eV}\right)$ just by simple structural isomerization.

To unravel such an interesting phenomenon of absorption properties, density functional theory (DFT) and time-dependent DFT (TD-DFT) calculations were carried out at the B3LYP/6$31(\mathrm{~d}, \mathrm{p})$ level. As shown in Fig. S2a, $\uparrow$ DTA-IC-S features a highly planar backbone with four 4-methylphenyl groups out of the plane, while DTA-IC-M exhibits a slightly twisted structure which might be due to the steric hindrance between $\mathrm{S}$ atoms in thiophene rings and hydrogens at 1 and 5 positions of anthracene. Fig. $2 \mathrm{c}$ and $\mathrm{d}$ reveal that the highest occupied molecular orbital (HOMO) is mainly localized on anthracene and fused thiophene rings for both DTA-IC-S and DTA-IC-M. However, a pronounced difference is observed for the lowest unoccupied molecular orbitals (LUMOs). The LUMO of DTA-IC$\mathrm{S}$ is mainly concentrated on electron-withdrawing ending groups with marginal delocalization on anthracene, while the LUMO of DTA-IC-M is fully delocalized along the conjugated backbone covering the anthracene and fused thiophene rings as well as ending groups, indicative of a more conjugated backbone for DTA-IC-M than that for DTA-IC-S. This is reasonable by considering that unavoidable cross-conjugation mode exists in DTA-IC-S as displayed in Fig. S2c $\dagger$ whereas no conjugation node is found for DTA-IC-M despite of its slightly twisted structure. ${ }^{12}$ Moreover, the upshifted HOMO level $(-5.32$ to $-5.25 \mathrm{eV})$ and downshifted LUMO level $(-3.21$ to $-3.50 \mathrm{eV})$ for DTA-IC-M suggest its narrower bandgap compared to DTA-IC-S. To further confirm the remarkable variation in absorption properties for these two isomers, the simulated absorption spectra were conducted by the TD-DFT calculation as shown in Fig. S2b. $\dagger$ Apparently, a large redshift of $\sim 140 \mathrm{~nm}$ is also observed from DTA-IC-S to DTA-IC-M which is in good agreement with the experimental results. Our findings suggest that structural isomerization employed in this work could furnish a simple and effective means to construct NNA with facile synthesis.

The electrochemical properties of DTA-IC-S and DTA-IC-M were investigated by cyclic voltammetry ${ }^{13}(\mathrm{CV})$ as shown in Fig. 3a. Distinctive oxidative and reductive behaviors could be detected for both compounds, and the HOMO/LUMO levels of $-5.73 /-3.83$ and $-5.53 /-4.05 \mathrm{eV}$ were derived for DTA-IC-S and DTA-IC-M, respectively, based on the onsets of the redox peaks. The trend of energy level variation is in accordance with that from the DFT calculations. In addition to the frontier molecular orbital (FMO) energy levels, the electron mobility $\left(\mu_{\mathrm{e}}\right)$ is also probed by the space-charge-limited-current (SCLC) method $^{14}$ (Fig. S3 $\uparrow$ and Table 1). A relatively lower $\mu_{\mathrm{e}}$ of $1.06 \times 10^{-6} \mathrm{~cm}^{2}$ $\mathrm{V}^{-1} \mathrm{~s}^{-1}$ is obtained for DTA-IC-M than that of DTA-IC-S (3.90 $\times$ $10^{-6} \mathrm{~cm}^{2} \mathrm{~V}^{-1} \mathrm{~s}^{-1}$ ) which may be attributed to the twisted backbone of DTA-IC-M and the resultant weaker molecular packing in neat film. 
Table 1 Optical properties, electronic energy levels and electron mobilities of DTA-IC-S and DTA-IC-M

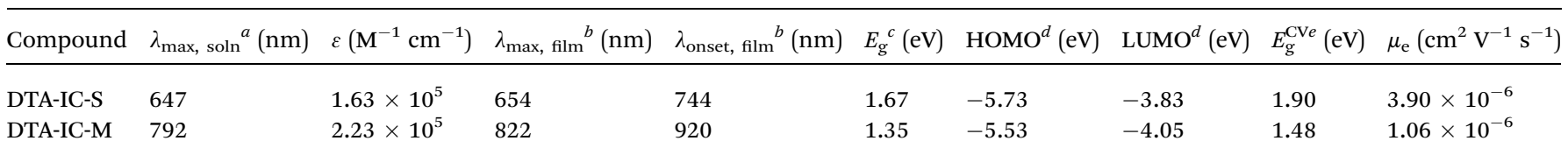

${ }^{a}$ Measured in chloroform solution with a concentration of $10^{-5} \mathrm{~mol} \mathrm{~L}^{-1} .{ }^{b}$ Measured in neat film. ${ }^{c}$ Estimated from the absorption edge in neat film using the equation: $E_{\mathrm{g}}=1243 / \lambda_{\text {onset }}(\mathrm{eV}) .{ }^{d}$ Measured in neat film using cyclic voltammetry $(\mathrm{CV}) .{ }^{e}$ Electrochemical bandgap from CV measurement.
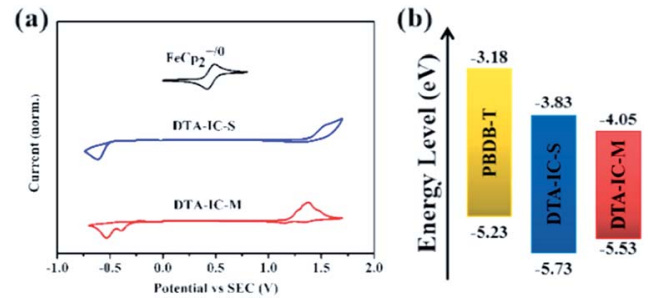

Fig. 3 (a) Cyclic voltammograms of DTA-IC-S and DTA-IC-M in acetonitrile with $0.1 \mathrm{M} \mathrm{Bu}_{4} \mathrm{NPF}_{6}$ as the supporting electrolyte; (b) energy level diagram of donor polymer PBDB-T and electron acceptors DTA-IC-S and DTA-IC-M.

To evaluate the potential of DTA-IC-M as a NNA, OSC devices are fabricated with the configuration of indium tin oxide (ITO)/ poly(3,4-ethylenedioxythiopene): poly(styrene sulfonate) (PEDOT: PSS)/active layer/amino $N$-oxide perylene diimide (PDINO) ${ }^{15} / \mathrm{Al}$, where PBDB-T ${ }^{16}$ is selected as the donor polymer. In addition, the acceptor DTA-IC-S is also used as a control. The optimized D: a weight ratio of PBDB-T: DTA-IC-S and PBDB-T: DTA-IC-M are $1: 1.5$ and $1: 1$, respectively, and no additives are needed during the device fabrication. The corresponding current density-voltage $(J-V)$ characteristics of the devices measured under $100 \mathrm{~mW} \mathrm{~cm}^{-2}$ simulated sunlight illumination are displayed in Fig. 4a. The DTAIC-S based device shows a PCE of $6.09 \%$, with a $V_{\text {oc }}$ of $0.94 \mathrm{~V}$, a $J_{\text {sc }}$ of $12.27 \mathrm{~mA} \mathrm{~cm}^{-2}$ and a FF of 0.529 , which is in consistent with the
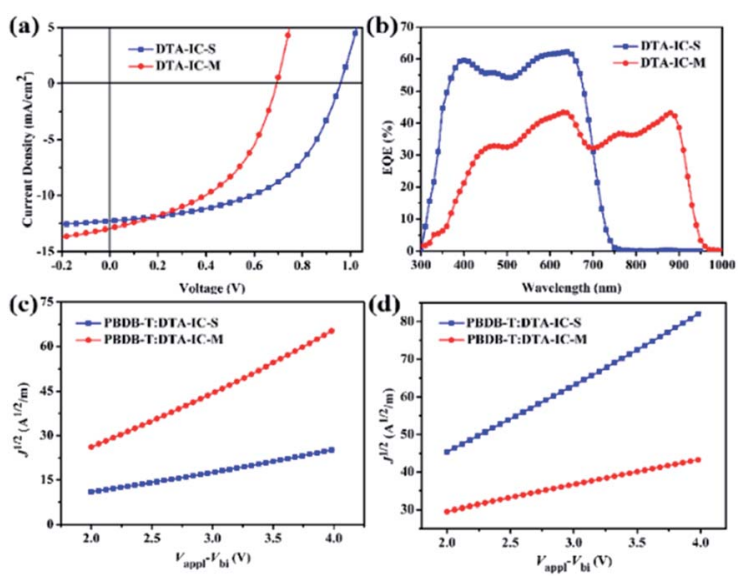

Fig. 4 (a) J-V characteristics of the optimized OSCs under the illumination of AM 1.5G, $100 \mathrm{~mW} \mathrm{~cm}^{-2}$; (b) EQE curves of the corresponding OSCs; (c) $J^{1 / 2}-V$ curves of the electron-only devices for the optimal active layer; (d) $J^{1 / 2}-V$ curves of the hole-only devices for the optimal active layer. result reported in our previous work. ${ }^{11}$ In contrast, the device based on DTA-IC-M shows a lower PCE of $4.20 \%$ which mainly resulted from the lower $V_{\mathrm{oc}}(0.69 \mathrm{~V})$ and $\mathrm{FF}(0.468)$. Despite of this, the slightly enhanced $J_{\mathrm{sc}}\left(12.96 \mathrm{~mA} \mathrm{~cm}{ }^{-2}\right)$ could be obtained from the DTA-IC-M based device which can be attributed to the additional NIR absorption of DTA-IC-M. As shown in Fig. 4b, the external quantum efficiency (EQE) spectra reveal that a much broader photo-to-current response from visible to NIR region (300 to 950 $\mathrm{nm}$ ) is achieved for the DTA-IC-M based device which is in good agreement with its red-shifted absorption in film state. Although the EQE value is generally lower for the DTA-IC-M based device than that of the DTA-IC-S based on in the visible region, the absorption contribution in the NIR region enables a higher integrated $J_{\mathrm{sc}}$ of $12.56 \mathrm{~mA} \mathrm{~cm}^{-2}$ for the DTA-IC-M based cell compared to $11.93 \mathrm{~mA} \mathrm{~cm}^{-2}$ for the DTA-IC-S base one, suggesting the great significance of NIR harvesting for such low bandgap electron acceptor.

To understand the lower PCE of the NNA DTA-IC-M compared to the medium bandgap acceptor DTA-IC-S, energy levels are taken into consideration first. As shown in Fig. 3b, the lower-lying LUMO of DTA-IC-M is responsible for the decreased $V_{\text {oc }}$ in the device compared to DTA-IC-S $(0.69 \mathrm{~V} v s .0 .94 \mathrm{~V}) .{ }^{17}$ However, the energy loss $\left(E_{\text {loss }}\right)$ is reduced to $0.66 \mathrm{eV}$ for the DTA-IC-M based device as compared to that of the DTA-IC-S based cell $(0.73 \mathrm{eV})$ according to the equation: $E_{\text {loss }}=E_{\mathrm{g}}^{\mathrm{opt}}-$ $e V_{\mathrm{oc}}{ }^{18}$ This may be ascribed to suppressed nonradiative recombination losses for electron acceptors with a low bandgap..$^{19}$ Subsequently, the charge transport properties in the blend films were investigated using the SCLC method as illustrated in Fig. $4 \mathrm{c}$ and $\mathrm{d}$ with the calculated data listed in Table S2. $\dagger$ The $\mu_{\mathrm{e}}$ is comparable for both DTA-IC-S and DTA-IC-M based films $\left(2.96 \times 10^{-5} \mathrm{~cm}^{2} \mathrm{~V}^{-1} \mathrm{~s}^{-1}\right.$ vs. $9.37 \times 10^{-5} \mathrm{~cm}^{2} \mathrm{~V}^{-1}$ $\left.\mathrm{s}^{-1}\right)$, whereas the hole mobility $\left(\mu_{\mathrm{h}}\right)$ of DTA-IC-M blend film $\left(1.16 \times 10^{-5} \mathrm{~cm}^{2} \mathrm{~V}^{-1} \mathrm{~s}^{-1}\right)$ is an order of magnitude lower than that of DTA-IC-S blend film $\left(1.96 \times 10^{-4} \mathrm{~cm}^{2} \mathrm{~V}^{-1} \mathrm{~s}^{-1}\right)$ which leads to a much more unbalanced $\mu_{\mathrm{e}} / \mu_{\mathrm{h}}$ (8.07) for DTA-IC-M based device and the resultant lower FF. This could be further confirmed by morphological analysis. Based on the atomic force microscopy (AFM) (Fig. S4 $\dagger$ ) and transmission electron microscopy (TEM) images (Fig. S5 $\dagger$ ), it can be seen that more obvious fiber-like aggregates exist in the PBDB-T:DTA-IC-S blend film with a larger root-mean-square (RMS) roughness of $3.12 \mathrm{~nm}$, while the PBDB-T:DTA-IC-M blend shows a more homogenous film with a RMS roughness of $1.39 \mathrm{~nm}$. This likely indicates that DTA-IC-M blend film suffers from an unsatisfactory phase separation which results in lower charge transporting abilities, increased charge recombination losses and 
the lower FF, ultimately. ${ }^{8 d}$ Similar results could also be obtained from two dimensional (2D) grazing-incidence wide-angle X-ray scattering (GIWAXS) (Fig. S6†). The weaker scattering along both in-plane and out-of-plane directions are observed for DTAIC-M compared to DTA-IC-S in their pristine films due to the more twisted molecular backbone of DTA-IC-M and undermining intermolecular stacking. Similarly, the PBDB-T:DTA-ICM blend film shows a slightly weaker $\pi-\pi$ scattering along outof-plane direction compared to the PBDB-T:DTA-IC-S blend film which may be owing to the more disruptive PBDB-T crystallization when blended with the DTA-IC-M with a lower ordering. Therefore, the nonplanar structure of DTA-IC-M is regarded as the main origin for the weaker packing, lower charge mobility, poorer phase separation and ultimately lower device performance in OSC devices. However, this issue can be tackled by further structural optimization, e.g. replacing fused thiophene units with furan units to reduce steric hindrance. Please note that the most significant finding in this work is the realization of a novel NIR electron acceptor featuring a simple structure and facile synthesis by a newly developed structural isomerization method.

Compared with several benchmark nonfullerene acceptors (Table S3 $\dagger$ ), the acceptor DTA-IC-M synthesized here displays a fairly narrow optical bandgap of $1.35 \mathrm{eV}$. It should be noted that the bandgap of the benchmark non-fullerene acceptors becomes narrower in principle as the field progresses (Table $\mathrm{S} 3 \dagger)$. To date, the best-performance non-fullerene acceptor Y6 has a bandgap of $1.33 \mathrm{eV},{ }^{1 h}$ which is highly comparable to that $(1.35 \mathrm{eV})$ of our acceptor. The results demonstrate the greatly advantage of DTA-IC-M for non-fullerene solar cells in terms of optoelectronic properties. We must admit that the performance of our acceptor is greatly lower than that of the state-of-the-art acceptors, which reflects the fact that there is a great room for further performance improvement through donor polymer pairing and film morphology optimization. Hence the isomerization method presented in this work provides an effective way (even if not universal) to develop novel NIR acceptors, which are highly pursued for maximizing the device performance of nonfullerene organic solar cells. In addition, developing novel NIR nonfullerene acceptors is essential for efficient ternary solar cells or tandem solar cells. More efforts on structural optimization using this isomerization method are currently undergoing and further improvement of PCE could be anticipated in the future.

In summary, a new NIR electron acceptor DTA-IC-M is developed via a structural isomerization method with simple chemical structure and facile synthesis. Compared to its medium bandgap isomeric acceptor DTA-IC-S, a remarkable bathochromic shift $(\sim 170 \mathrm{~nm})$ is attained for the new DTA-IC$\mathrm{M}$, which shows a maximum absorption peak over $800 \mathrm{~nm}$ and a narrowed bandgap of $1.35 \mathrm{eV}$. Due to the enhanced phototo-current response in the NIR region, a higher $J_{\mathrm{sc}}$ of $12.96 \mathrm{~mA}$ $\mathrm{cm}^{-2}$ is achieved for the DTA-IC-M based solar cell with a PCE of $4.21 \%$. Moreover, the universality of this isomerization approach and the isomerization effect on the stability are currently undergoing in our lab and further PCE enhancement could be anticipated in the near future.

\section{Conflicts of interest}

There are no conflicts to declare.

\section{Acknowledgements}

The authors gratefully acknowledge the financial support from the National Natural Science Foundation of China (no. 21805128 and 21774055) and Shenzhen Science and Technology Innovation Commission (JCYJ20170817105905899). H. Y. W. is grateful to the financial support from the National Research Foundation of Korea (2016M1A2A2940911). This work is also partially supported by Center for Computational Science and Engineering of Southern University of Science and Technology.

\section{Notes and references}

1 (a) Y. Lin and X. Zhan, Mater. Horiz., 2014, 1, 470-488; (b) S. Li, L. Ye, W. Zhao, S. Zhang, S. Mukherjee, H. Ade and J. Hou, Adv. Mater., 2016, 28, 9423-9429; (c) W. Zhao, D. Qian, S. Zhang, S. Li, O. Inganäs, F. Gao and J. Hou, Adv. Mater., 2016, 28, 4734-4739; (d) J. Zhao, Y. Li, G. Yang, K. Jiang, H. Lin, H. Ade, W. Ma and H. Yan, Nat. Energy, 2016, 1, 15027; (e) G. C. Bazan, Sci. China: Chem., 2017, 60, 1109-1110; (f) J. Hou, O. Inganäs, R. H. Friend and F. Gao, Nat. Mater., 2018, 17, 119; (g) B. Fan, D. Zhang, M. Li, W. Zhong, Z. Zeng, L. Ying, F. Huang and Y. Cao, Sci. China: Chem., 2019, 62, 746-752; (h) J. Yuan, Y. Zhang, L. Zhou, G. Zhang, H. L. Yip, T. K. Lau, X. Lu, C. Zhu, H. Peng, P. A. Johnson, M. Leclerc, Y. Cao, J. Ulanski, Y. Li and Y. Zou, Joule, 2019, 3, 1140-1151.

2 (a) H. Sun, B. Liu, C. W. Koh, Y. Zhang, J. Chen, Y. Wang, P. Chen, B. Tu, M. Su, H. Wang, Y. Tang, Y. Shi, H. Y. Woo and X. Guo, Adv. Funct. Mater., 2019, 1903970; (b) Y. Wang, Z. Yan, M. A. Uddin, X. Zhou, K. Yang, Y. Tang, B. Liu, Y. Shi, H. Sun, A. Deng, J. Dai, H. Y. Woo and X. Guo, Sol. $R R L$, 2019, 3, 1900107; (c) K. Yang, Q. Liao, C. W. Koh, J. Chen, M. Su, X. Zhou, Y. Tang, Y. Wang, Y. Zhang, H. Y. Woo and X. Guo, J. Mater. Chem. A, 2019, 7, 9822-9830.

3 H. Li, Z. Xiao, L. Ding and J. Wang, Sci. Bull., 2018, 63, 340342.

4 L. Meng, Y. Zhang, X. Wan, C. Li, X. Zhang, Y. Wang, X. Ke, Z. Xiao, L. Ding, R. Xia, H. L. Yip, Y. Cao and Y. Chen, Science, 2018, 361, 1094.

5 R. Yu, H. Yao, Y. Cui, L. Hong, C. He and J. Hou, Adv. Mater., 2019, 31, 1902302.

6 (a) X. Li, T. Yan, H. Bin, G. Han, L. Xue, F. Liu, Y. Yi, Z. Zhang, T. P. Russelle and Y. Li, J. Mater. Chem. A, 2017, 5, 2258822597; (b) Y. Lin and X. Zhan, Adv. Energy Mater., 2015, 5, 1501063; (c) S. Dai, F. Zhao, Q. Zhang, T. K. Lau, T. Li, K. Liu, Q. Ling, C. Wang, X. Lu, W. You and X. Zhan, J. Am. Chem. Soc., 2017, 139, 1336-1343; (d) B. Jia, S. Dai, Z. Ke, C. Yan, W. Ma and X. Zhan, Chem. Mater., 2018, 30, 239-245.

7 S. Dai, T. Li, W. Wang, Y. Xiao, T. K. Lau, Z. Li, K. Liu, X. Lu and X. Zhan, Adv. Mater., 2018, 30, 1706571.

8 (a) X. Guo and M. D. Watson, Org. Lett., 2008, 10, 5333-5336; (b) K. H. Hendriks, W. Li, M. M. Wienk and R. A. Janssen, J. 
Am. Chem. Soc., 2014, 136, 12130-12136; (c) X. Guo, Q. Liao, E. F. Manley, Z. Wu, Y. Wang, W. Wang, T. Yang, Y. E. Shin, X. Cheng, Y. Liang, L. X. Chen, K. J. Baeg, T. J. Marks and X. Guo, Chem. Mater., 2016, 28, 2449-2460; (d) H. Yao, Y. Chen, Y. Qin, R. Yu, Y. Cui, B. Yang, S. Li, K. Zhang and J. Hou, Adv. Mater., 2016, 28, 8283-8287; (e) J. Sun, X. Ma, Z. Zhang, J. Yu, J. Zhou, X. Yin, L. Yang, R. Geng, R. Zhu, F. Zhang and W. Tang, Adv. Mater., 2018, 30, 1707150; $(f)$ Y. Cui, C. Yang, H. Yao, J. Zhu, Y. Wang, G. Jia, F. Gao and J. Hou, Adv. Mater., 2017, 29, 1703080; $(g)$ J. Sun, X. Ma, Z. Zhang, J. Yu, J. Zhou, X. Yin, L. Yang, R. Geng, R. Zhu, F. Zhang and W. Tang, Adv. Mater., 2018, 30, 1707150.

9 (a) Z. X. Liu, T. K. Lau, G. Zhou, S. Li, J. Ren, S. K. Das, R. Xia, G. Wu, H. Zhu, X. Lu, H. L. Yip, H. Chen and C. Z. Li, Nano Energy, 2019, 63, 103807; (b) F. X. Chen, J. Q. Xu, Z. X. Liu, M. Chen, R. Xia, Y. Yang, T. K. Lau, Y. Zhang, X. Lu, H. L. Yip, A. K. Y. Jen, H. Chen and C. Z. Li, Adv. Mater., 2018, 30, 1803769; (c) H. Xu, Y. Yang, C. Zhong, X. Zhan and X. Chen, J. Mater. Chem. A, 2018, 6, 6393-6401.

10 F. Liu, Z. Zhou, C. Zhang, J. Zhang, Q. Hu, T. Vergote, F. Liu, T. P. Russell and X. Zhu, Adv. Mater., 2017, 29, 1606574.

11 Y. Wang, B. Liu, C. W. Koh, X. Zhou, H. Sun, J. Yu, K. Yang, H. Wang, Q. Liao, H. Y. Woo and X. Guo, Adv. Energy Mater., 2019, 9, 1803976.
12 (a) M. Gholami and R. R. Tykwinski, Chem. Rev., 2006, 12, 4997-5027; (b) G. W. P. van Pruissen, J. Brebels, K. H. Hendriks, M. M. Wienk and R. A. J. Janssen, Macromolecules, 2015, 8, 2435-2443.

13 (a) J. Wang, J. Zhang, Y. Xiao, T. Xiao, R. Zhu, C. Yan, Y. Fu, G. Lu, X. Lu, S. R. Marder and X. Zhan, J. Am. Chem. Soc., 2018, 140, 9140-9147; (b) S. Dai, Y. Xiao, P. Xue, J. James Rech, K. Liu, Z. Li, X. Lu, W. You and X. Zhan, Chem. Mater., 2018, 30, 5390-5396.

14 P. N. Murgatroyd, J. Phys. D: Appl. Phys., 1970, 3, 151-156.

15 Z. G. Zhang, B. Qi, Z. Jin, D. Chi, Z. Qi, Y. Li and J. Wang, Energy Environ. Sci., 2014, 7, 1966-1973.

16 D. Qian, L. Ye, M. Zhang, Y. Liang, L. Li, Y. Huang, X. Guo, S. Zhang, Z. a. Tan and J. Hou, Macromolecules, 2012, 45, 9611-9617.

17 Z. Ding, X. Long, C. Dou, J. Liu and L. Wang, Chem. Sci., 2016, 7, 6197-6202.

18 W. Li, K. H. Hendriks, A. Furlan, M. M. Wienk and R. A. Janssen, J. Am. Chem. Soc., 2015, 137, 2231-2234.

19 Y. Cui, H. Yao, J. Zhang, T. Zhang, Y. Wang, L. Hong, K. Xian, B. Xu, S. Zhang, J. Peng, Z. Wei, F. Gao and J. Hou, Nat. Commun., 2019, 10, 2515. 